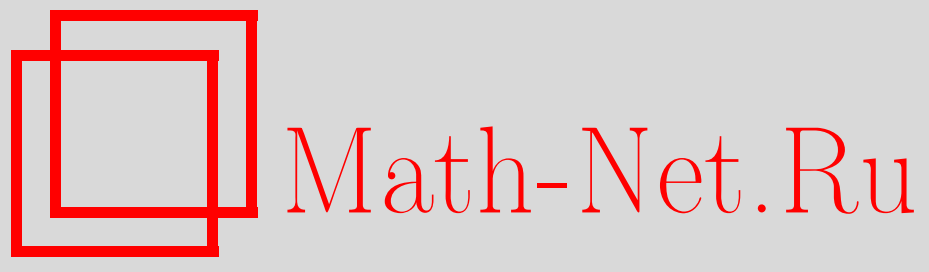

Е. Д. Лившиц, О равномерной аппроксимации на подмножествах, Матем. заметки, 2015, том 98, выпуск $5,797-800$

DOI: https://doi.org/10.4213/mzm10783

Использование Общероссийского математического портала Math-Net.Ru подразумевает, что вы прочитали и согласны с пользовательским соглашением http://www . mathnet.ru/rus/agreement

Параметры загрузки:

IP : 54.197 .217 .227

26 апреля 2023 г., 03:32:54

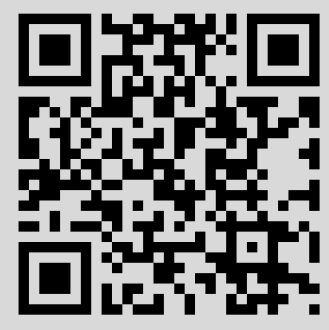




\section{О равномерной аппроксимации на подмножествах}

\section{Е. Д. Лившиц}

1. Развитие многих областей теории приближения было обусловлено необходимостью решения прикладных задач. В настоящей заметке рассматривается задача теории приближения, являющаяся формализацией задачи "аппроксимации фона фотографии документа" из теории обработки изображений [1]. Пусть заданы множество $X$ с вероятностной мерой $\mu$, множество функций $M \subset \mathcal{L}_{\infty}(X)$ и число $u \in(0,1)$. В теории обработки изображений делается предположение, что для $X=[0,1]^{2}$, класса $M$, состоящего из достаточно гладких функций, и функции $f \in L_{\infty}(X)$, соответствующей фотографии документа, имеет место представление

$$
f=m+f_{0}+e, \quad m \in M, \quad f_{0}, e \in \mathcal{L}_{\infty}(X),
$$

в котором $\mu\left(\operatorname{supp} f_{0}\right)<u$, и величина $\epsilon:=\|e\|_{\infty}$ достаточно мала. Тогда гладкая функция $m$ будет соответствовать яркости фона, $f_{0}$ с малым носителем - собственно тексту документа, а $e$ - малому равномерному шуму. В этом случае преобразование $f \rightarrow\left(f-m_{0}-\epsilon\right)_{+}$ сделает фон фотографии равномерно белым и обеспечит желаемое улучшение качества изображения.

Для $A \subset X$ и $p, 1 \leqslant p \leqslant \infty$, будем использовать следующее обозначение:

$$
\|f\|_{p, A}:=\left\|f \chi_{A}\right\|_{p},
$$

где $\chi_{A}$ - индикатор множества $A$. Для того, чтобы перейти к постановке задачи, определим

$$
|f|_{u}:=\inf _{A \subset X, \mu(A) \geqslant 1-u}\|f\|_{\infty, A}, \quad \operatorname{Dst}_{u}(f, M):=\inf _{m \in M}|f-m|_{u} .
$$

В наиболее общем виде задача состоит в вычислении $\operatorname{Dst}_{u}(f, M)$ и нахождении $m$, на котором эта величина достигается или почти достигается. Для $|\cdot|_{u}$ не выполняется неравенство треугольника, поэтому вычисление $\operatorname{Dst}_{u}(f, M)$ является невыпуклой задачей. В настоящей работе мы исследуем эффективные алгоритмы ее приближенного решения, т.е. алгоритмы нахождения таких

$m \in M$, для которых величина $|f-m|_{u}$ была бы достаточно близка к $\operatorname{Dst}_{u}(f, M)$.

То, что слагаемое $f_{0}$, несущее информацию о тексте, сосредоточено на множестве малой меры $\left(\mu\left(\operatorname{supp} f_{0}\right)<\epsilon\right)$ указывает, что рассматриваемый вопрос можно отнести к задаче о сжатом измерении. Как известно, одними из наиболее эффективных способов решения задачи о сжатых измерениях являются $l_{1}$ минимизация и жадные алгоритмы [2], [3]. Рассмотрим их аналоги для решения задачи (2).

$l_{1}$-МинимизАция. В качестве решения задачи (2) выбирается

$$
P_{1}(f):=\operatorname{argmin}_{m \in M}\|f-m\|_{1} .
$$

ЖАДНЫЙ АЛГОРИТм ВЫБОРА ПОДМНОЖЕСТвА. Пусть задано $p, 1 \leqslant p<\infty$. Построим последовательность вложенных подмножеств $\varnothing=U_{0}^{p} \subset U_{1}^{p} \subset \cdots$ и элементов $G_{k}^{p}(f) \in M$, $k \geqslant 0$. Пусть $U_{0}^{p}:=\varnothing$. Для каждого $k \geqslant 0$ определим

$$
\begin{aligned}
G_{k}^{p} & :=G_{k}^{p}(f):=\operatorname{argmin}_{m \in M}\|f-m\|_{p, X \backslash U_{k}^{p} .} \\
U_{k+1}^{p} & :=U_{k}^{p} \cup\left\{x \in X \backslash U_{k}^{p}:\left|f(x)-G_{k}^{p}(x)\right|>\frac{1}{2}\left\|f-G_{k}^{p}\right\|_{\infty, X \backslash U_{k}^{p}}\right\} .
\end{aligned}
$$

С точки зрения скорости вычисления наибольший интерес представляет случай $p=2$.

Исследование выполнено за счет гранта Российского научного фонда (проект № 14-50-00005).

DOI: $10.4213 / \mathrm{mzm} 10783$

(C) Е. Д. Лившиц, 2015 
ЗАмечАниЕ 1. В обоих этих определениях мы предполагаем, что $\operatorname{argmin}(\cdot)$ достигается на каком-то элементе из $M$; в противном случае необходимо рассмотреть почти оптимальное решение.

ЗАмечАние 2. Пусть $M:=L_{n}$ является чебышевской системой функций и $\left|f-m_{0}\right|_{u}=$ 0 , при некотором $m_{0} \in M$. Тогда у задачи (2) есть вероятностное решение, значительно превосходящее детерминисткие подходы. Выберем случайным образом числа $x_{i} \in X, 1 \leqslant$ $i \leqslant n$, найдем $l \in L_{n}$, для которой $l\left(x_{i}\right)=f\left(x_{i}\right), 1 \leqslant i \leqslant n$, и проверим является ли найденная $l$ решением задачи, т.е. верно ли, что $|f-l|_{u}=0$. Легко проверить, что вероятность того, что за первые $N$ проверок решение не будет найдено, не превышает $\exp (-c N)$.

В следующих двух разделах рассматривается "модельная ситуация", когда

$$
\left|f-m_{0}\right|_{u}=0, \quad m_{0} \in M .
$$

2. Следующая простая лемма вытекает из неравенства треугольника и является стандартной в теории $l_{1}$-минимизации.

Лемма. Пусть множество $M$ является линейным подпространством $\mathcal{L}_{\infty}(X)$. Предположим, что для любого $U \subset X, \mu(U) \leqslant u$ июбого $m \in M, m \neq 0$, выполняется неравенство

$$
\|m\|_{1, U}<\|m\|_{1, X \backslash U}, \quad m \neq 0 .
$$

Тогда для любого $f \in \mathcal{L}_{\infty}(X)$ из равенства $\left|f-m_{0}\right|_{u}=0, m_{0} \in M$, будет вытекатъ равенство $P_{1}(f)=m_{0}$.

ДоКАзАТЕЛЬСТво. Без ограничения общности можно предположить, что $m_{0}=0$; тогда найдется $U \subset X, \mu(U) \leqslant u$, для которого

$$
f(x)=0, \quad x \in X \backslash U .
$$

По неравенству треугольника и определению $l_{1}$-минимизации

$$
\begin{gathered}
\left\|f-P_{1}(f)\right\|_{1, U}+\left\|P_{1}(f)\right\|_{1, U} \geqslant\|f\|_{1, U} \stackrel{(4)}{=}\|f\|_{1} \geqslant\left\|f-P_{1}(f)\right\|_{1} \\
=\left\|f-P_{1}(f)\right\|_{1, X \backslash U}+\left\|f-P_{1}(f)\right\|_{1, U} \stackrel{(4)}{=}\left\|P_{1}(f)\right\|_{1, X \backslash U}+\left\|f-P_{1}(f)\right\|_{1, U}, \\
\left\|P_{1}(f)\right\|_{1, U} \geqslant\left\|P_{1}(f)\right\|_{1, X \backslash U} .
\end{gathered}
$$

Из неравенства (3) непосредственно вытекает, что $P_{1}(f)=0=m_{0}$.

СледствиЕ. Пусть $X$ является одномерным тором $\mathcal{T}$, а $M$ - множеством тригонометрических полиномов $T_{n}$, степени не выше $n$. Тогда найдется такое $C>0$, что для функиии $f \in \mathcal{L}_{\infty}(\mathcal{T}), \operatorname{Dst}_{u}\left(f, T_{n}\right)=0, u \leqslant c n^{-1}$, метод $l_{1}$-минимизации будет давать точное решение: $\left|f-P_{1}(f)\right|_{u}=0$.

Выполнение неравенства (3) для тригонометрических полиномов вытекает из неравенства С. М. Никольского [4], точное значение константы $c$ было недавно найдено Ю. В. Малыхиным и К. С. Рютиным [5].

3. Перейдем к исследованию скорости сходимости жадного алгоритма выбора подмножества. Хотя, как отмечалось с выше, с вычислительной точки зрения наибольший интерес представляет случай $p=2$, мы проведем рассуждения для всех $p, 1 \leqslant p<\infty$. Пусть $M=$ $L_{n}$ является линейным подпространством, для которого выполняется неравенство Никольского

$$
\|l\|_{\infty} \leqslant C_{N} n^{1 / p}\|l\|_{p}, \quad l \in L_{n}
$$

где константа $C_{N}$ может зависеть от $p$. В частности $M$ может быть множеством тригонометрических полиномов степени не выше заданной. (В этом случае $C_{N}=3$, см. [4].) 
Теорема 1. Пусть для $M=L_{n}$ выполняется неравенство Никольского (5). Если

$$
\left|f-m_{0}\right|_{u}=0, \quad f \in \mathcal{L}_{\infty}(X), \quad m_{0} \in L_{n},
$$

$u$

$$
u \leqslant \frac{C_{N}^{-p}}{12 n}
$$

то для всех $k \in \mathbb{N}$ будет выполняться оченка

$$
\left|f-G_{k}^{p}(f)\right|_{u} \leqslant \frac{1}{6}\left\|f-m_{0}\right\|_{\infty}\left(\frac{3}{4}\right)^{k} .
$$

ДокАЗАТЕЛЬСтво. Без ограничения общности можно считать, что $m_{0}=0$, т.е.

$$
f(x)=0, \quad x \in X \backslash U, \quad \mu(U) \leqslant u .
$$

Для сокращения обозначений будем писать $q$ вместо $G_{k}^{p}=G_{k}^{p}(f)$ и $U_{k}$ вместо $U_{k}^{p}$. Докажем по индукции, что для $k \geqslant 0$ будут выполняться следующие утверждения:

$$
U_{k+1} \subset U, \quad\|f\|_{\infty, U \backslash U_{k+1}} \leqslant \frac{3}{4}\|f\|_{\infty, U \backslash U_{k}} .
$$

По определению жадного алгоритма выбора подмножества

$$
\|f-q\|_{p, X \backslash U_{k}} \leqslant\|f-0\|_{p, X \backslash U_{k}} .
$$

Следовательно,

$$
\|q\|_{p, X \backslash U}^{p} \stackrel{(7)}{=}\|f-q\|_{p, X \backslash U}^{p} \leqslant\|f-q\|_{p, X \backslash U_{k}}^{p} \leqslant\|f\|_{p, X \backslash U_{k}}^{p} \stackrel{(7)}{=}\|f\|_{p, U \backslash U_{k}}^{p} \leqslant\|f\|_{\infty, U \backslash U_{k}}^{p} \mu(U) .
$$

Применим неравенство Никольского к $q \in L_{n}$ :

$$
\begin{aligned}
\|q\|_{\infty}^{p} & \leqslant C_{N}^{p} n\|q\|_{p}^{p} \leqslant C_{N}^{p} n\left(\|q\|_{p, X \backslash U}^{p}+\|q\|_{p, U}^{p}\right) \\
& \leqslant C_{N}^{p} n\left(\|f\|_{\infty, U \backslash U_{k}}^{p} \mu(U)+\|q\|_{\infty}^{p} \mu(U)\right) \stackrel{(7)}{\leqslant} C_{N}^{p} n u\left(\|f\|_{\infty, U \backslash U_{k}}^{p}+\|q\|_{\infty}^{p}\right) .
\end{aligned}
$$

Отсюда и неравенства (6) будет следовать оценка

$$
\|f-q\|_{\infty, X \backslash U}=\|q\|_{\infty, X \backslash U} \leqslant\|q\|_{\infty} \leqslant \frac{1}{6}\|f\|_{\infty, U \backslash U_{k}} .
$$

Тогда будут иметь место следующие неравенство:

$$
\|f-q\|_{\infty, X \backslash U} \leqslant \frac{1}{6}\|f\|_{\infty, U \backslash U_{k}} \leqslant \frac{1}{3}\|f-q\|_{\infty, X \backslash U_{k}} .
$$

Следовательно, $U_{k+1} \subset U$. Если $x \in U \backslash U_{k}$ и $|f(x)|>3 / 4\|f\|_{\infty, U \backslash U_{k}}$, то

$$
|f(x)-q(x)|>\frac{3}{4}\|f\|_{\infty, U \backslash U_{k}}-\|q\|_{\infty} \stackrel{(10)}{\geqslant} \frac{1}{2}\left(\|f\|_{\infty, U \backslash U_{k}}+\|q\|_{\infty}\right) \geqslant \frac{1}{2}\|f-q\|_{\infty, U \backslash U_{k}} .
$$

Поэтому

$$
\left\{x \in U \backslash U_{k}:|f(x)|>\frac{3}{4}\|f\|_{\infty, U \backslash U_{k}}\right\} \subset U_{k+1},
$$

что доказывает индукционные предположения (8). В результате получаем

$$
\begin{aligned}
\left|f-G_{k}^{p}(f)\right|_{u} & \leqslant\left\|f-G_{k}^{p}(f)\right\|_{\infty, X \backslash U}=\|f-q\|_{\infty, X \backslash U} \stackrel{(7)}{=}\|q\|_{\infty, X \backslash U} \\
& \leqslant\|q\|_{\infty} \stackrel{(10)}{\leqslant} \frac{1}{6}\|f\|_{\infty, U \backslash U_{k}} \stackrel{(8)}{\leqslant} \frac{1}{6}\|f\|_{\infty}\left(\frac{3}{4}\right)^{k} \leqslant \frac{1}{6}\left\|f-m_{0}\right\|_{\infty}\left(\frac{3}{4}\right)^{k} .
\end{aligned}
$$


4. Даже в модельном случае $\operatorname{Dst}_{u}(f, M)=0$ положительный результат удается получить только когда $f$ отличается от $l \in L_{n}$ на множестве меры порядка $n^{-1}$. С точки зрения задачи выравнивания фона это условие является слишком ограничительным. С другой стороны, в этой задаче естественно ограничить сверху производную фона - элементов множества $M$ :

$$
M \subset W_{\infty}^{1}([0,1]), \quad\left\|m^{\prime}\right\|_{\infty} \leqslant C_{0} \quad \text { или } \quad M \subset W_{\infty}^{1}\left([0,1]^{2}\right), \quad\|\nabla m\|_{\infty} \leqslant C_{0} .
$$

Эти соотношения позволяют оценить сверху $\|\cdot\|_{\infty}$ через $\|\cdot\|_{p}$. Легко проверить, что можно выбрать константы $c_{1}, c_{2}$ со следующим свойством: для любого $m \in M$ и $U \subset X, \mu(U) \leqslant$ $\min \left(c_{1}\|m\|_{\infty}, 1 / 2\right)$, выполняется неравенство

$$
\|m\|_{p, X \backslash U} \geqslant c_{2} \min \left(\|m\|_{\infty}, 1\right)^{(p+1) / p} .
$$

В этой ситуации удается показать, что жадный алгоритм выбора подмножества позволяет исключить из аппроксимации те точки, где функция $f$ сильно отличалась от оптимального $m_{0} \in M$ и $G_{k}^{p}(f)$ являются достаточно хорошим приближением к $f$. Более того, в этой ситуации допустим шум порядка $u^{(p+1) / p}$.

Tеорема 2. Существуют такие константы $C_{i}>1,1 \leqslant i \leqslant 4$, зависящие только от $C_{0}$, что если для $f \in \mathcal{L}_{\infty}(X), m_{0} \in M u u \in(0,1 / 2]$ будет выполнятвся неравенство

$$
\left|f-m_{0}\right|_{u}<C_{1} u^{(p+1) / p},
$$

mo $\partial \Omega я$

$$
k_{0}=\left[C_{2}\left(\ln \left(\left\|f-m_{0}\right\|_{\infty}\right)_{+}-\ln u+1\right)\right]
$$

будет справедлива оценка

$$
\left|f-G_{k_{0}}^{p}\right|_{u} \leqslant C_{4} C_{3}^{p} u .
$$

Автор выражает глубокую благодарность члену-корреспонденту РАН профессору С. В. Конягину за плодотворное обсуждение результатов настоящей работы.

\section{СПИСОК ЦИТИРОВАННОЙ ЛИТЕРАТУРЫ}

[1] Gabriel de França Pereira e Silva, Rafael Dueire Lins, André Ricardson Silva, Image Analysis and Recognition, Lecture Notes in Computer Science, 7950, Springer-Verlag, Berlin, 2013, 290-298. [2] D. L. Donoho, IEEE Trans. Inform. Theory, 52:4 (2006), 1289-1306. [3] Е. Д. Лившиц, Матем. сб., 203:2 (2012), 33-44. [4] С. М. Никольский, Приближение функиий многих переменных и теоремы вложения, Наука, М., 1977. [5] Ю. В. Малыхин, К. С. Рютин, Матем. сб., 205:11 (2014), 95-124.

Е. Д. Лившиц

Математический институт им. В.А. Стеклова

Российской академии наук

E-mail: livshitz@rambler.ru
Поступило

23.04.2015 